\title{
Risk Factors Associated with the Requirement for Pharmaceutical Intervention in the Hospital Setting: A Systematic Review of the Literature
}

\author{
Emma Suggett $^{1} \cdot$ John Marriott ${ }^{2}$
}

Published online: 25 July 2016

(c) The Author(s) 2016. This article is published with open access at Springerlink.com

\begin{abstract}
Background A number of methods exist for the risk assessment of hospital inpatients to determine the likelihood of patients experiencing drug-related problems (DRPs), including manual review of a patient's medication (medication reviews) and more complex electronic assessment using decision support alerts in electronic prescribing systems. A systematic review was conducted to determine the evidence base for potential risks associated with adult hospital inpatients that could not only lead to medication-related issues but might also be directly associated with pharmacist intervention.

Objectives The aims were to perform a systematic review of the literature in order to (1) identify all measurable risk factors associated with adult hospital inpatients that potentially lead to a pharmaceutical intervention; (2) critically evaluate the quality of the identified research; and (3) further subcategorise potential risk factors, so that pharmaceutical services may be targeted to patients "at risk" by identifying potential risk factors in a patient's electronic hospital record.
\end{abstract}

Methods A systematic review, conducted in June 2013, searched ten medical literature databases for all papers identifying risks leading to pharmacist interventions or DRPs, adverse drug events (ADEs), adverse drug reactions,

Emma Suggett

emma.suggett@uhb.nhs.uk

1 Pharmacy Department, University Hospitals Birmingham NHS Foundation Trust, Queen Elizabeth Hospital Birmingham, University of Birmingham, Mindelsohn Way, Edgbaston, Birmingham B15 2WB, UK

2 School of Pharmacy, College of Medical and Dental Sciences, University of Birmingham, Birmingham, UK drug errors (where not included in the definition of an ADE), and medication-related problems. The search identified 7720 titles, from which 120 papers were sourced. A hand search of a further 11 journals was also performed. No date restrictions were imposed. All primary research and literature reviews were included. Summary articles were excluded with the exception of literature reviews. The inclusion of search outputs was validated by a third party pharmacy research graduate.

Results From the 7720 titles, 38 publications met the inclusion criteria for the review. The ten most frequently reported risk factors associated with medication-related issues that may potentially lead to a hospital pharmaceutical intervention are as follows (ranked in descending order of frequency): prescription of certain drugs or classes of drugs, polypharmacy, elderly patients (defined as over 65 years), female gender, poor renal function, the presence of multiple comorbidities, length of patient stay, history of drug allergy or sensitivity, patient compliance issues, and poor liver function. The ten classes of drugs most frequently reported to be associated with medication-related issues leading to a hospital pharmaceutical intervention are as follows (ranked in descending order of frequency): intravenous antimicrobials, thrombolytics/anticoagulants, cardiovascular agents, central nervous system agents, corticosteroids, diuretics, chemotherapy, insulin/hypoglycaemics, opiates, and anti-epileptics.

Conclusion Review of the literature identified 38 papers, from which the ten most frequently reported risk factors linked with factors that are potentially associated with hospital pharmaceutical interventions (all definitions included) were identified. No papers were identified that demonstrated a direct causal relationship between a potential risk factor and hospital pharmaceutical interventions. All of the potential risk factors associated with 
problems with the use of medicines can be identified from patient records on admission to hospital. These risk factors may be used to identify patients at risk, with a view to targeting pharmaceutical intervention in order to minimise risks of problems with medicines and improve efficiency of clinical pharmacy services.

\section{Key Points}

A total of 38 papers identified the ten most frequently reported measurable risk factors for medication-related issues (all international definitions included), all of which may be identified from hospital inpatient records.

Twenty-eight of these papers identified the ten most frequently reported drugs or classes of drug associated with medication-related issues; further work is required to quantify these risks.

No papers discussed the risk factors associated with the requirement for pharmacist intervention. This may be because of poor evidence for an association of pharmacist interventions with a reduction in medicines-related incidents.

\section{Introduction}

\subsection{Background}

In recent years, there has been an increasing drive to improve the quality of care delivered by the United Kingdom's (UK's) National Health Service (NHS), whilst at the same time improving productivity and efficiency. Quality, Innovation, Productivity and Prevention (QIPP) is a national policy seeking to reduce costs in the NHS through reduction in readmission to hospital. Several studies have aimed to determine the risk factors leading to hospital admission, and these studies are valuable in meeting the QIPP agenda [1].

It has been shown that more than $6 \%$ of all hospital admissions are due to issues related to medication [2] prior to admission, with the result that there have been an increasing number of community-based methodologies trialled internationally to identify and target patients with risk factors for intervention [3-8]. Most of these studies have used incident report review, prescription chart review, direct observation or trigger tools to identify at-risk patients. The trigger tool method, in which patients are screened for perceived risk factors for medication problems, has been shown to be the most effective and labour-efficient method for identifying vulnerable patients [9]. In some cases, the use of electronic prescribing systems (EPs) and clinical decision support (CDS) has resulted in the development of a number of "trigger tools" driven by rule-based alerts programmed into a CDS system [10-12].

The purpose of this review was to determine the evidence base for problems associated with medicines after admission to hospital and to identify factors directly determining the most vulnerable patients requiring targeted intervention by a pharmacist. The intention would be to determine if these risk factors could be identified from the patient's medical notes (potentially electronic medical notes where an EP or CDS system is in place) to assist in targeting pharmacist intervention in order to improve the quality and efficiency of clinical pharmacy services.

Pharmacists will generally intervene in the case of medication problems inclusive of all definitions. As such, the review included and sought to make comparison between studies using all terminologies and definitions of issues related to drug treatment [such as adverse drug events (ADEs), adverse drug reactions (ADRs), drug errors, drug-related problems (DRPs), and medication-related problems (MRPs)]. However, in addition to targeting problems associated with specific drugs or regimens, pharmacists may intervene for reasons generally associated with non-clinical patient characteristics such as communication difficulties, confusion, or their refusal to comply with recommended treatment. Just as the risks associated with drug errors may differ slightly from those associated with ADRs, we should not assume that the risks associated with pharmacist intervention are identical to those leading to medicines-related issues. Any reviews identifying risks directly associated with the requirement for clinical pharmacist intervention were also included.

A number of corporate approaches to assess the risks associated with drug usage in the hospital setting already exist. Retrospective assessment of incident reports in the UK is widespread, with the majority of hospitals identifying local trends in drug-related incidents. Reporting ADRs to the Medicines and Healthcare Regulatory Agency (MHRA) and drug-related incidents to the National Patient Safety Agency (NPSA) [now replaced by NHS England (NHSE)] has been routine practice for many years, with the result that there is an increased awareness of high-risk prescribing amongst pharmacists and prescribers. The NPSA and now NHSE have issued alerts [13] pertaining to high-risk drugs, drug omissions, patients who are nil by mouth, and the administration of medicines using syringe drivers. Similarly, the Institute for Safe Medicines Practices (ISMP) in the USA [14] and the Australian Commission on Safety and Quality in Health Care (ACSQHC) [15] have used a similar system of alerts, raising awareness 
of the risk associated with medicines in organisations internationally.

This increasing awareness of risk in hospitals has led researchers in the UK to pilot a national trigger tool, the Medication Safety Thermometer [16]. The tool aims to direct pharmacists by identifying patients at risk of harm following the omission of high-risk drugs. These high-risk drugs were defined using data pooled from reports to the NPSA. This may prove to be a valuable tool in reducing patient harm, but does not address all of the risks that a pharmacist should target. Certain forms of risk reduction with direct clinical interventions to patients deemed to be "at risk" have developed as roles of the UK hospital pharmacist have evolved. Patients with polypharmacy, those with impaired renal or liver function, and those taking anti-epileptics or medication for Parkinson's disease may already be in receipt of increased pharmacist monitoring albeit on a qualitative ad hoc basis. Assessment of the impact of pharmaceutical intervention is also difficult since intervention is usually a preventative action, which influences the measurement of patient outcomes. The result is that the value of clinical pharmacy services is not well documented, communicated, or perceived by hospital managers in the UK.

Inpatients are unlikely to be rationally documented as "high risk" or in need of targeted intervention by a pharmacist with the possible exception of cases where a pharmaceutical care plan has been employed. However, producing a pharmaceutical care plan is extremely labour intensive and is therefore often only completed for complex cases in most hospitals in the UK. National initiatives such as the reduction of dosage omissions and targeting high-risk drugs and supporting patient adherence are increasingly taking up the time of clinical pharmacists. In a risk driven, resource-limited environment, targeting clinical pharmacy services to ensure safe, timely, high-quality services centred on patient safety should be paramount. To identify all patients who require clinical pharmacist intervention and therefore target valuable pharmacy resources, we must identify all of the drug-related risks associated with the patient and their treatment.

\subsection{Aims}

The purpose of this systematic review was to determine the evidence base for measurable risk factors that pre-dispose patients to the requirement for a clinical pharmacist intervention in their treatment.

The intention is to use these outcomes in further research to build an evidence-based trigger tool, targeting individuals at risk of experiencing a problem with their medicines while in hospital. Risk scores are being increasingly researched with a view to targeting high-risk patients $[17,18]$; however, the intention for future research is to develop a score that encompasses all definitions of drug-related issues to direct pharmacy services.

The key aims were to search the international literature to:

1. Identify measurable risk factors for medicines-related issues that may signal the necessity for a pharmacist intervention.

2. Document the frequency of such risk factors.

3. Identify those risk factors that could be accessed from a patient's medical notes.

\subsection{Objectives}

The objectives are to:

1. Search for and document all primary research or literature reviews identifying measurable risk factors that can be associated with problems associated with medicines or the requirement for a clinical pharmacist intervention.

2. Critically evaluate the quality of the identified research through intensive reading.

3. Further subcategorise the identified risk factors to enable their identification or measurement in a patient's electronic hospital record.

\section{Methods}

The systematic review was carried out using the principles and checklist set out in the PRISMA statement [19]. Figure 1 outlines the methodology and summarises results at each stage of the review.

\subsection{Eligibility Criteria}

The PICOS method [20] was used to formulate the review question and identify free-text search terms through a combination of mind-mapping by E.S. and information from a focus group consisting of ten members of the UK West Midlands Clinical Pharmacy Group.

\subsubsection{Paper Inclusion Criteria}

Inclusion criteria included quantifiable risk factors, patients over 16 years, inpatients in secondary or tertiary care centres, inpatients in medical and surgical wards, all definitions of DRPs, ADEs, ADRs, MRPs, and clinical pharmacy interventions (defined as "The process of a pharmacist identifying, and making a recommendation in an attempt to prevent or resolve, a drug-related issue"; the 


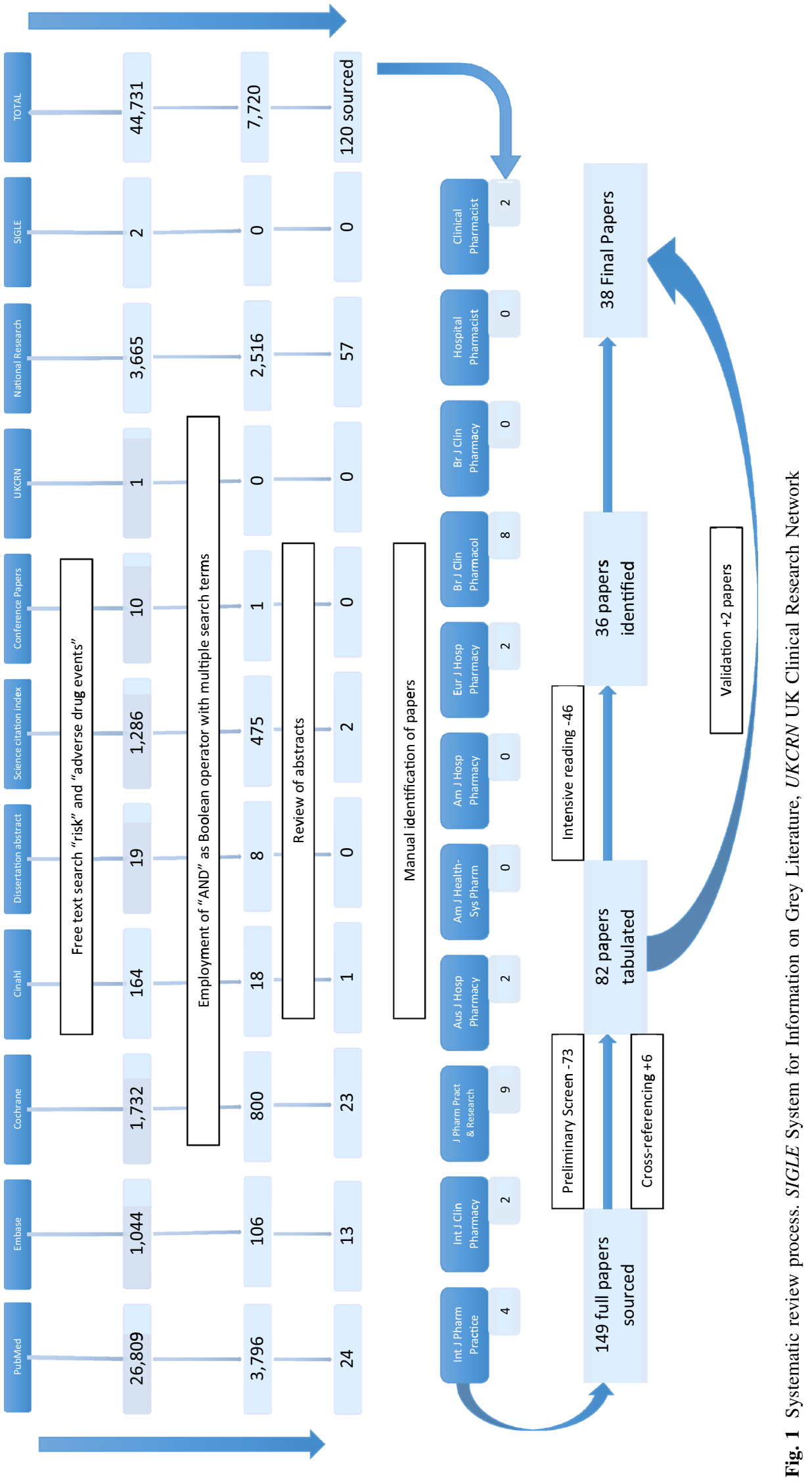


definition of an intervention does not include a routine, pharmacist review of medication without recommendation for a change in the patient's treatment), all primary research, and systematic reviews.

\subsubsection{Paper Exclusion Criteria}

Exclusion criteria included qualitative risk factors (e.g. patient's previous knowledge of medicines), studies reporting outcomes indirectly associated with pharmacist interventions or adverse events associated with medicines, e.g. medicines adherence, studies of patients 16 years or younger, outpatients, ambulatory care and communitybased studies, studies solely in patients in specialist care settings, e.g. intensive care, summary articles (with the exception of systematic reviews), and discussion articles.

\subsubsection{Search Terms}

Free-text search terms comprised the following: risk, risk assessment, clinical risk, susceptibility, drug, medicine, medicines reconciliation, drug history, clinical check, age, elderly, adult, compliance aids, medicines adherence, comorbidity/ies, long term conditions, therapeutic drug monitoring (TDM), renal function, liver function, prescription, early warning score, dose/dosage, pharmacy review, biochemistry, urea and electrolytes, tests, microbiology, intervention, adverse drug event, adverse drug reaction, drug error, medication error, and pharmacy service.

MeSH descriptors were identified from free-text terms inputted into the databases listed below and comprised the following: risk, risk factor, hospital risk, risk assessment, lifestyle risk reduction, risk reduction, clinical prediction rule, clinical prediction, health risk, health risk appraisal, pharmaceutical preparations, medicine, drug administration schedule, drug administration routes, drug combinations, drug hypersensitivity, drug interactions, drug synergism, drug therapy drug toxicity, medical history taking, drug prescriptions, decision support techniques, clinical pharmacy, medical informatics, pharmacists, pharmacy service hospital, pharmacy service, hospital, clinical pharmacy information systems, drug utilization review, pharmaceutical services, intervention studies, pharmacy service, hospital and medication errors.

\subsection{Information Sources}

Initially online searches were conducted in databases $1-10$ included in the list below.

Following the database search, a manual search was conducted of journals 11-21, using online access.
Databases searched:

1. MEDLINE-http://www.ncbi.nlm.nih.gov/PubMed

2. EMBASE-http://www.embase.com

3. Cochrane Data Base of Systematic Reviews-http:// www.cochrane.co.uk/en/index.html

4. CINAHL-http://www.cinahl.com

5. Dissertation Abstracts-http://www.umi.com/en-US/ catalogs/databases/detail/pqdt.shtml

6. Science Citation Index-http://thomsonreuters.com/ en/products-services/scholarly-scientific-research/ scholarly-search-and-discovery/web-of-science-corecollection.html

7. Conference Papers Index-http://ca2.csa.com/ factsheets/cpi-set-c.php

8. UK Clinical Research Network: Portfolio Databasehttp://public.ukcrn.org.uk

9. National Research Register Archive-http://www. nihr.ac.uk/Pages/NRRArchive.aspx

10. SIGLE (System for Information on Grey Literature)—http://www.opengrey.eu.

Journals manually searched:

11. Clinical Pharmacist-http://www.pharmpress.com/ product/13527967/clinical-pharmacist

12. Hospital Pharmacist_http://www.pharmj.com/ backissues/hp.html

13. British Journal of Clinical Pharmacy-http://www. clinicalpharmacy.org.uk/home

14. British Journal of Clinical Pharmacology-http:// onlinelibrary.wiley.com/journal/10.1111/(ISSN)13652125

15. European Journal of Hospital Pharmacy-http:// ejhp.bmj.com/

16. American Journal of Hospital Pharmacy — currently without website accessed at: https://www. researchgate.net

17. American Journal of Health-System Pharmacyhttp://www.ajhp.org/content/by/year

18. Australian Journal of Hospital Pharmacy-currently without website accessed at: http://search. informit.com.au/browseJournalTitle;res=IELHEA; issn $=0310-6810$

19. Journal of Pharmacy Practice and Researchhttp://search.informit.com.au/browseJournalTitle; res=IELHEA;issn $=1445-937 \mathrm{X}$

20. International Journal of Clinical Pharmacy (known as Pharmacy World and Science Prior to 2011) http://www.springer.com/medicine/internal/journal/ 11096

21. International Journal of Pharmacy Practice - http:// onlinelibrary.wiley.com/journal/10.1111/(ISSN)20427174. 


\subsection{Study Selection}

Between April and June 2013, searches were undertaken in chronological order using the same search terms listed above for all databases.

No date or language restrictions were applied during the review. However, the search was closed in July 2013 and identified no papers prior to 1966 . Further online searching for additional papers not already identified was conducted for grey literature using the free-text search terms listed above and, in particular, for internet publications linked to pharmaceutical interventions, using Google and Firefox as search engines.

After screening the abstracts, all potentially relevant full-text publications were evaluated through intensive reading by E.S. Citations included in the retrieved articles were reviewed and, if relevant, were sourced, evaluated, and the citations checked.

All sourced articles were tabulated to allow validation of a final list of citations and for a final list of included papers to be drawn up. The validation of this final list was carried out by an independent pharmacy research graduate who, using the agreed inclusion/exclusion criteria, evaluated the articles against their respective abstracts. Where the abstract did not provide sufficient information for the article to be evaluated against the inclusion/exclusion criteria, the full text was provided to the research graduate.

Finally, the research graduate and E.S. met to discuss any remaining articles to resolve disagreements; it was not necessary to resort to a third party reviewer as disagreements were resolved.

In order to quantify the results of the review, thematic analysis was undertaken. Through intensive reading, risks were identified as such as those listed as independent risk factors in the research conclusions and subsequently tabulated to allow for common themes (risks) to be identified.

\subsection{Data Collection Process}

Included risk factors were not required to have been shown to be a statistically significant independent risk factor, although where reported statistical methods excluded or included a risk factor as an independent risk factor, this was noted in the conclusions.

\subsection{Synthesis of Results}

The association of risk factors was noted in the results table. A "negative" association was noted where the research had shown:

1. No association between the potential risk factor and issues related to the use of medicines or the requirement for a pharmacist intervention;
2. The potential risk factor was not an independent risk factor for issues related to the use of medicines or the requirement for a pharmacist intervention; or

3. The potential risk factor was a protective factor for problems associated with the use of medicines or the requirement for a pharmacist intervention.

The frequencies of positive and negative associations with risk factors were documented in Table 1 in order to identify the most frequently reported risk factors.

All risk factors identified in the literature by more than one primary research paper were listed under their respective description and all others noted as "other".

Those studies that demonstrated an association between certain drugs or drug classes and problems with their use were further tabulated (Table 2) to identify these "highrisk" drugs.

\section{Results}

Figure 1 summarises the publication outputs at each stage of the review process.

Using search terms "risk" and "adverse drug events", 44,731 articles were identified initially from online searches. This was reduced to 7720 through the use of "AND" as the Boolean operator to link to a third relevant search term.

All resulting titles were viewed and 120 abstracts identified for possible inclusion in the review from searching online search engines.

A further 29 full-text papers were identified from manual searching of journals 11-21.

The search of Google and Firefox provided no additional references. In total 149 full texts were sourced.

Preliminary screening of the paper abstracts and crossreferencing of the citation by E.S. identified a resulting 82 papers, which were tabulated and independently evaluated.

Intensive reading of the resultant 82 papers eliminated a further 46 in accordance with the study inclusion/exclusion criteria.

The same 46 publications were independently eliminated by the research graduate, while two papers of the 82 were included back into the final results after discussion and agreement with the primary author.

The resulting 38 papers (including four literature reviews) were tabulated in Table 1 and intensive reading identified any potential risk factors.

Ten risk factors were identified in more than one research paper (in descending order of prevalence): prescription of certain drugs or classes of drugs, polypharmacy, elderly patients (defined as over 60-75 years or older), female gender, poor renal function, the presence of 


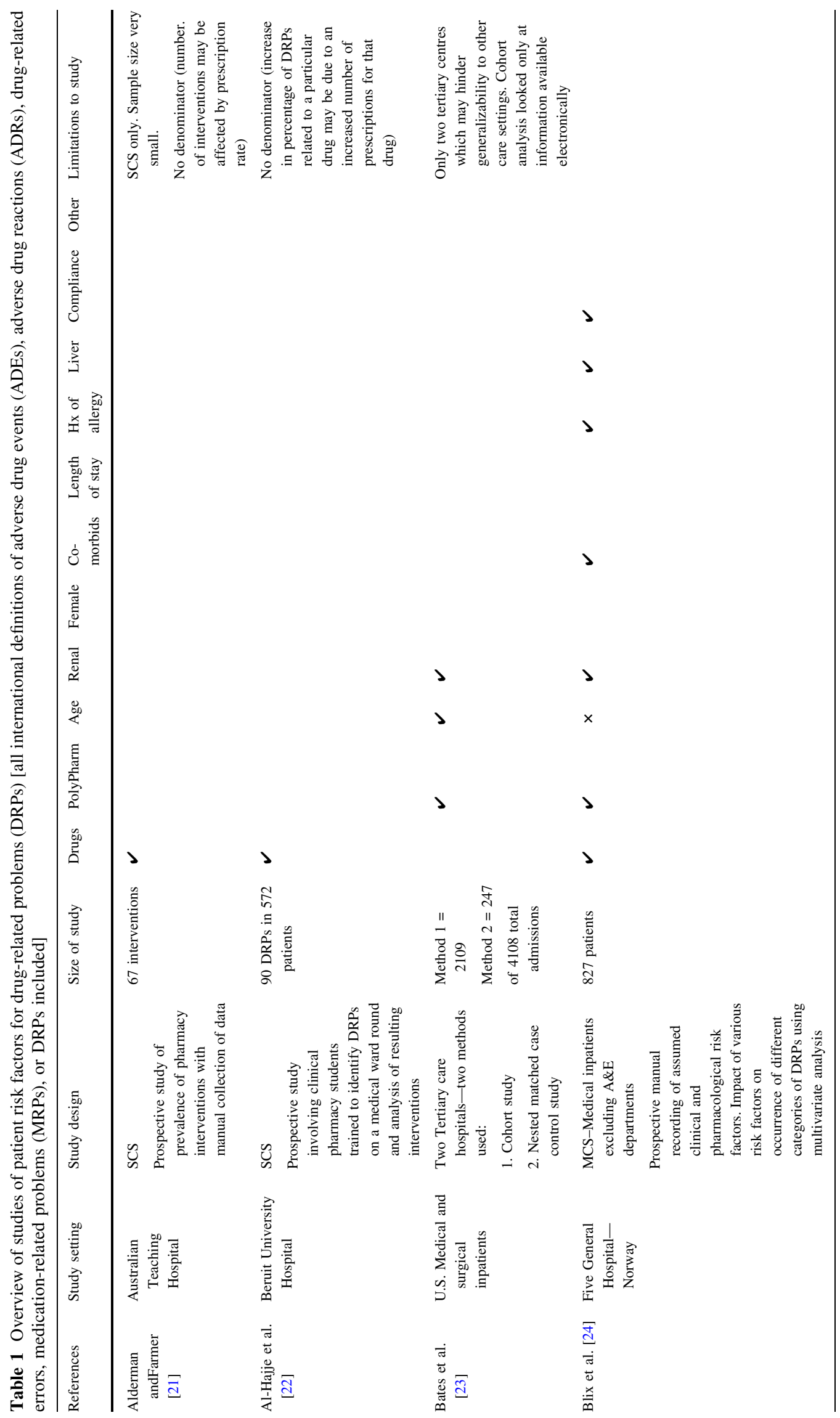




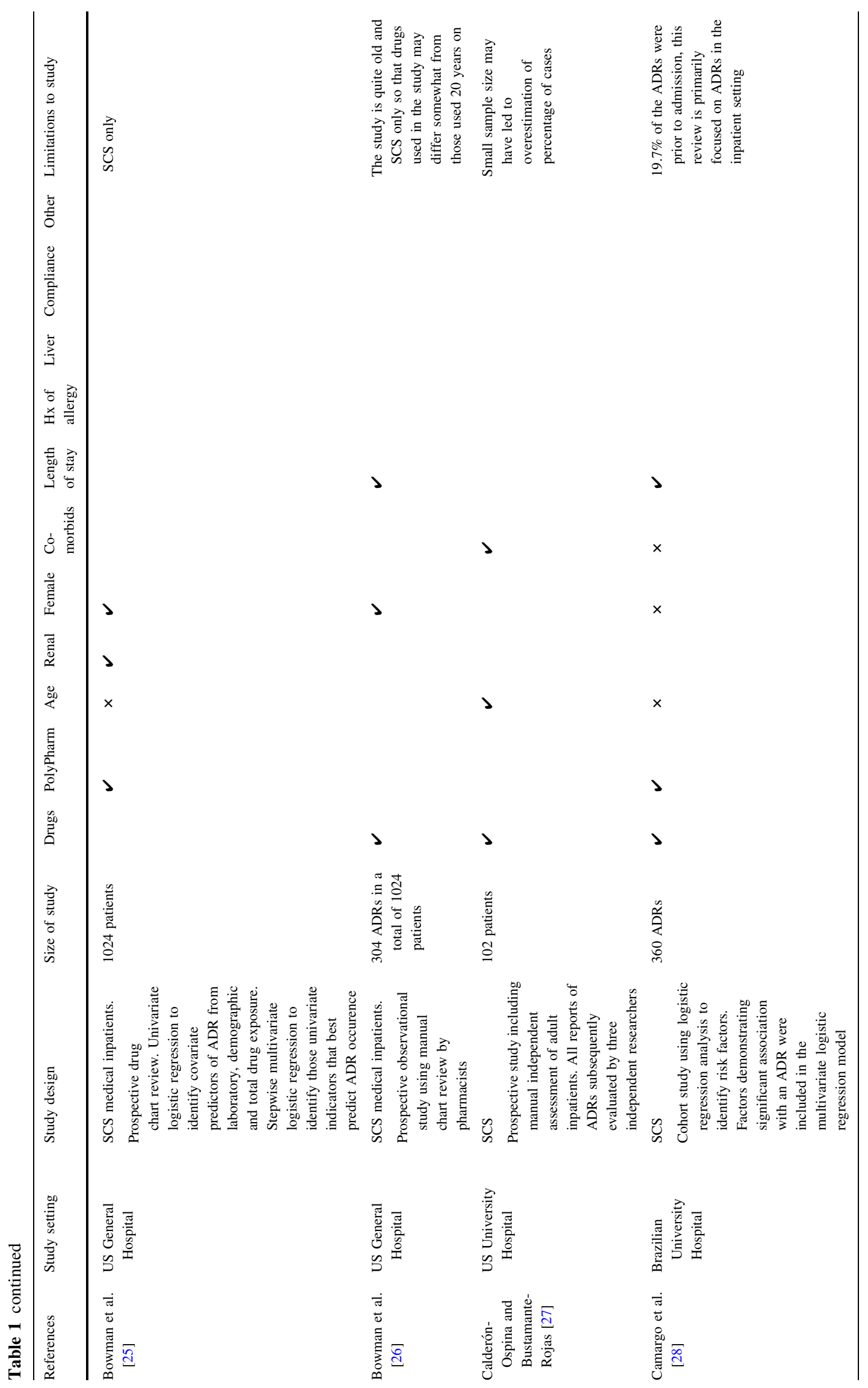




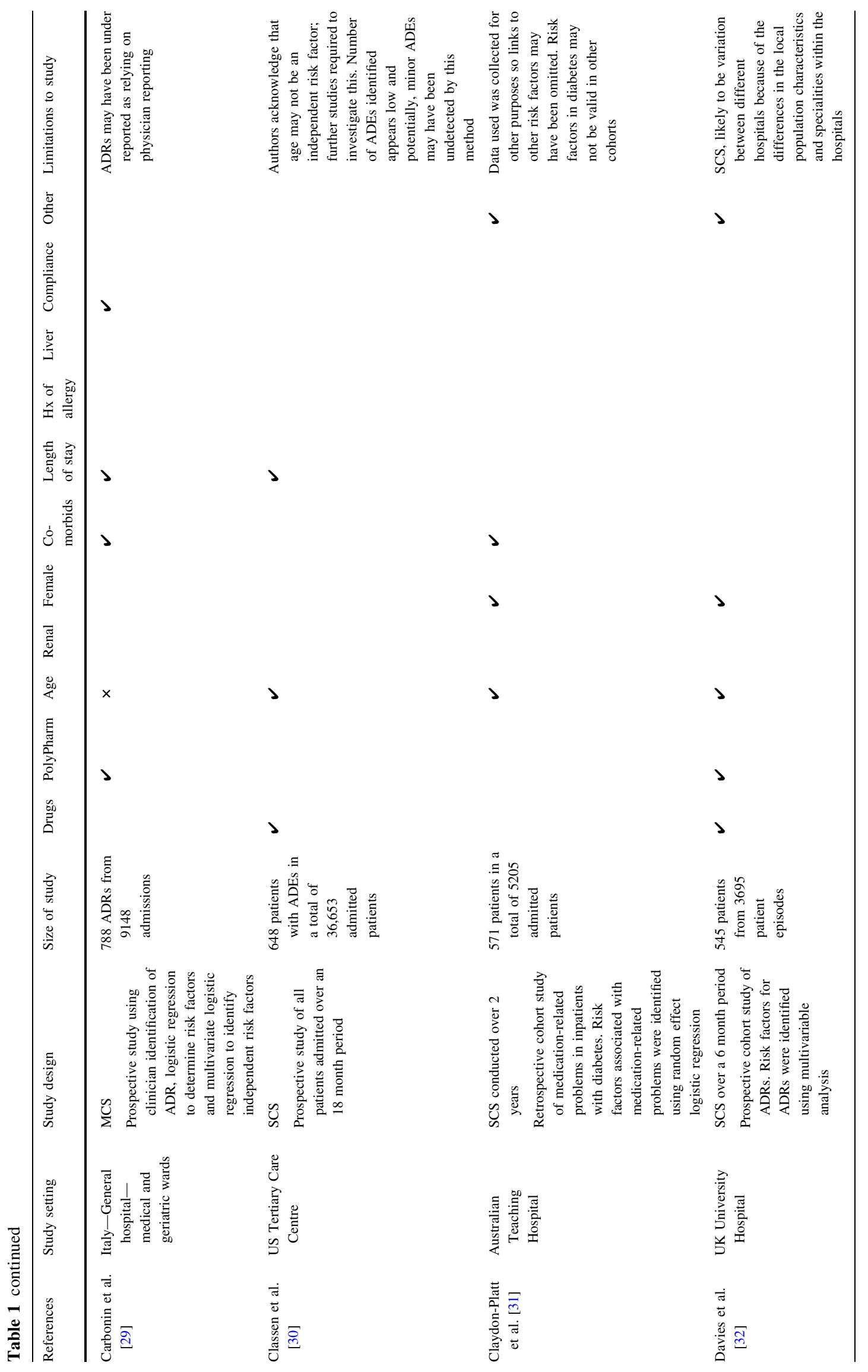




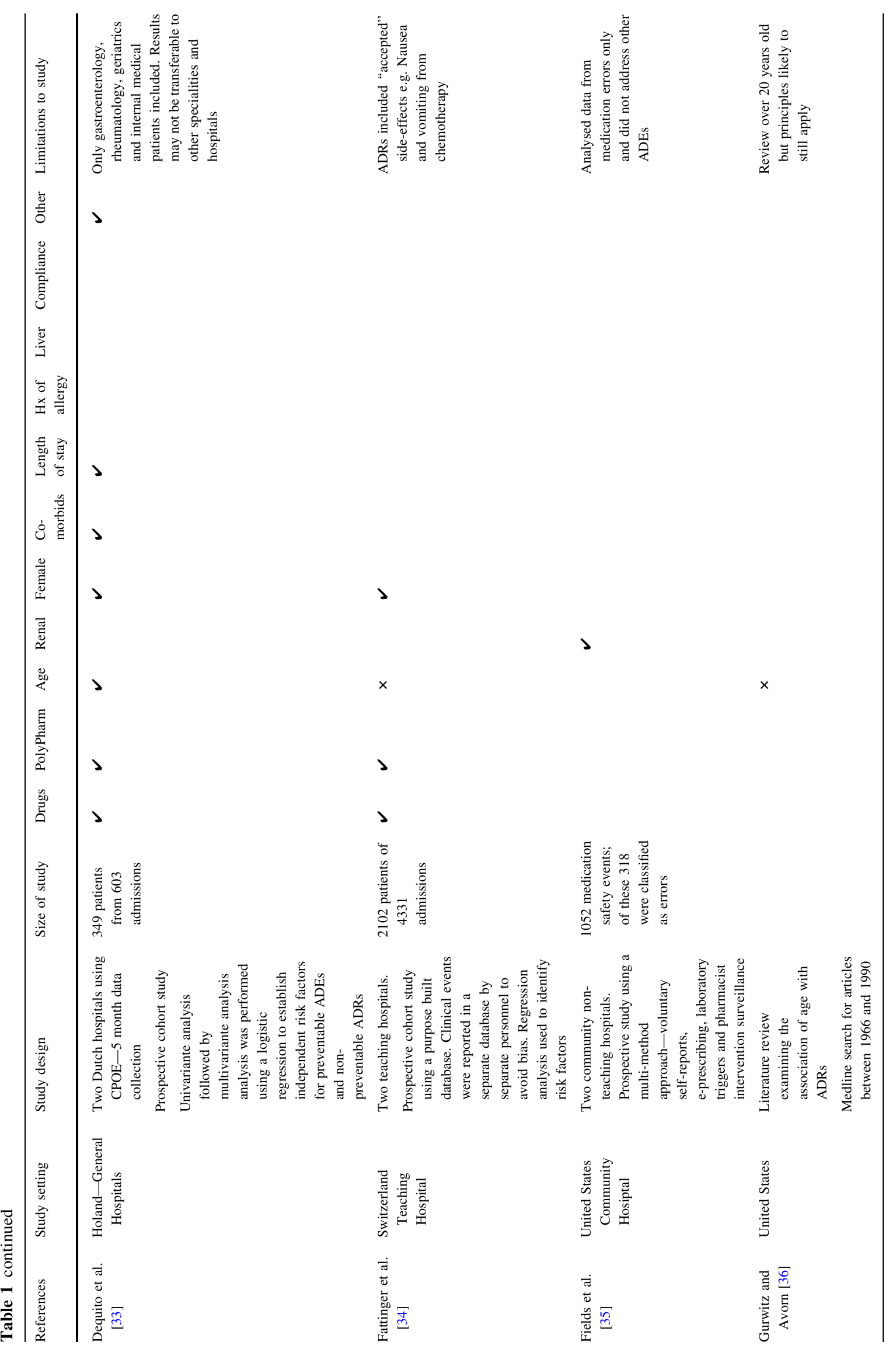




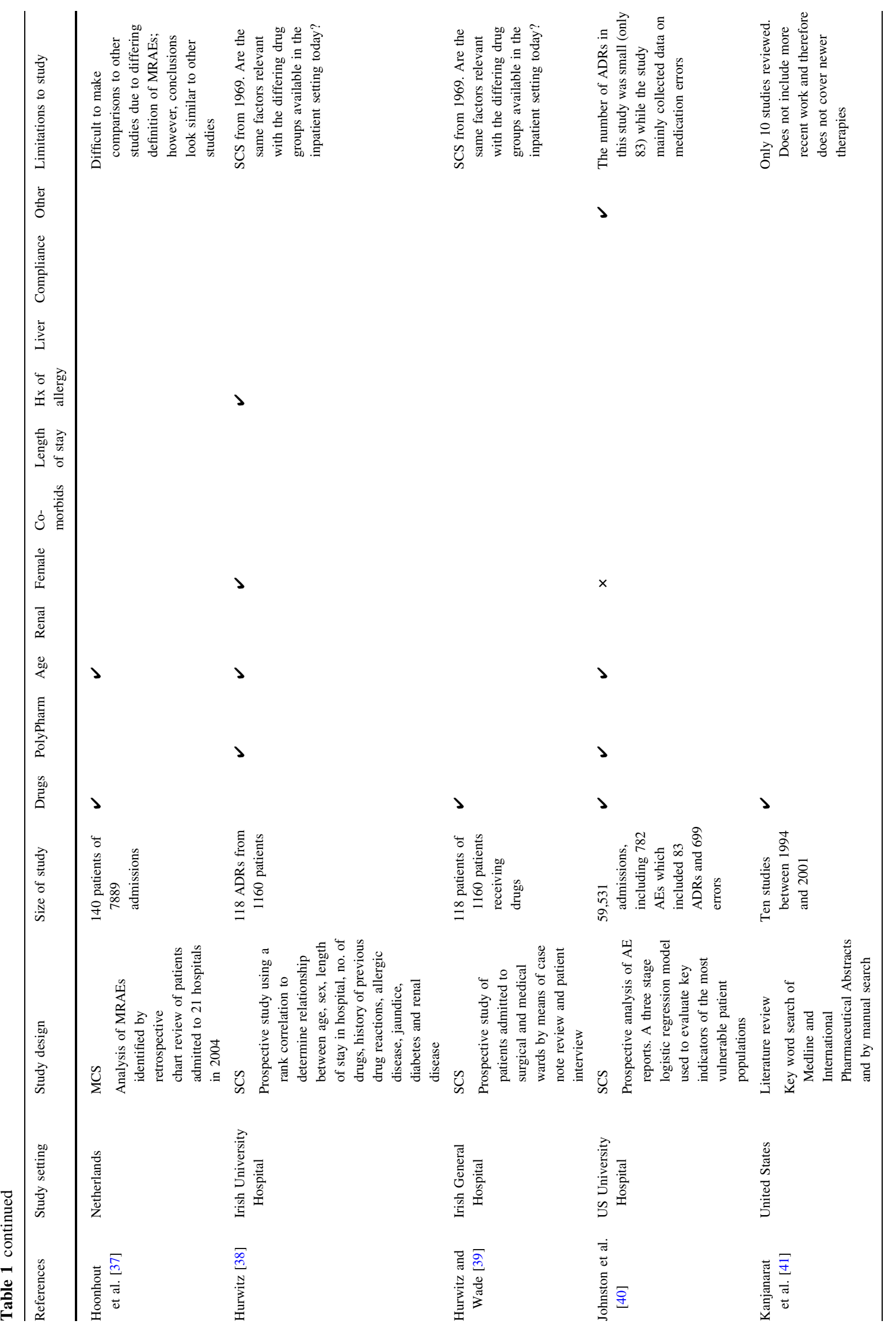




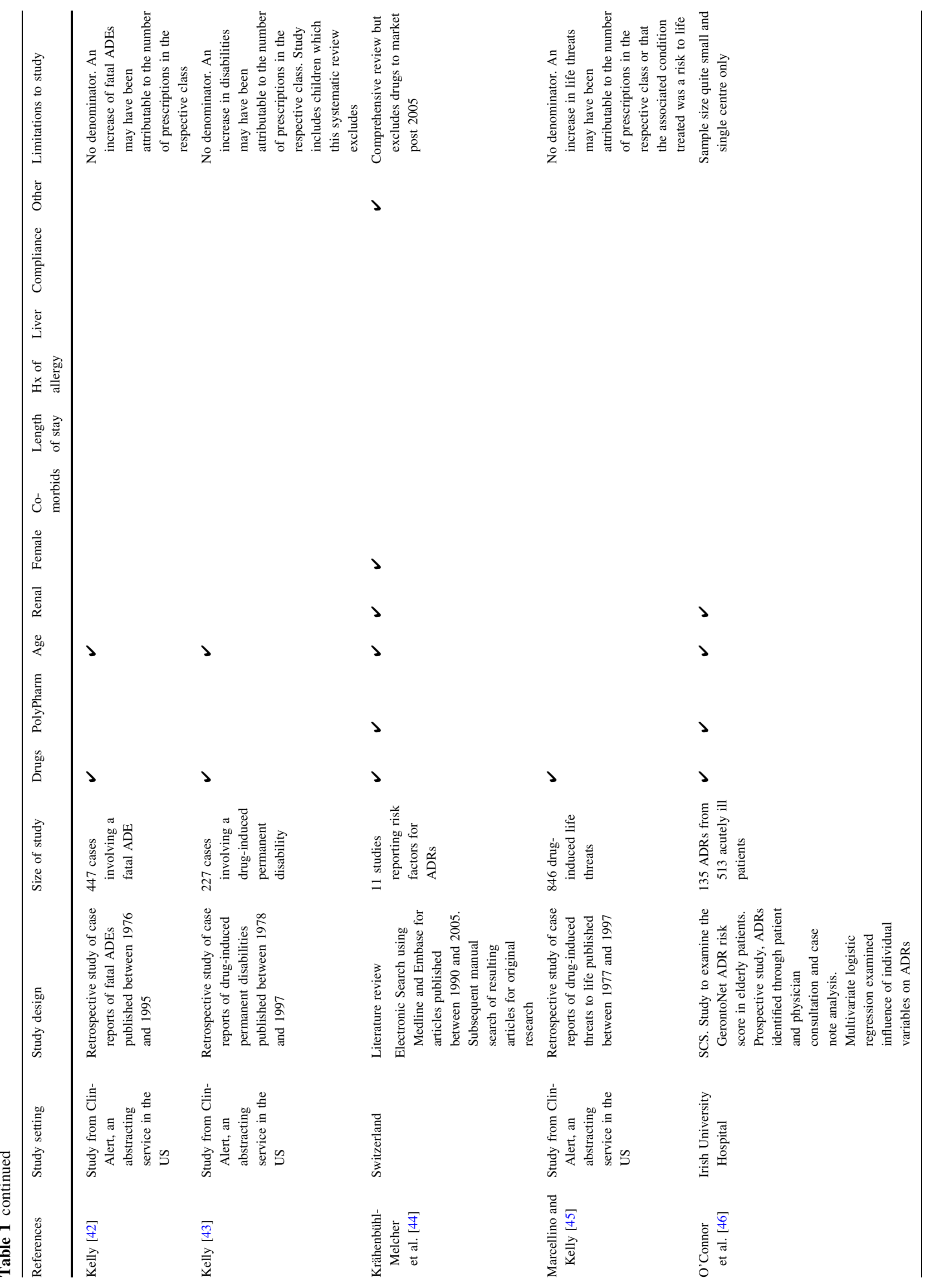




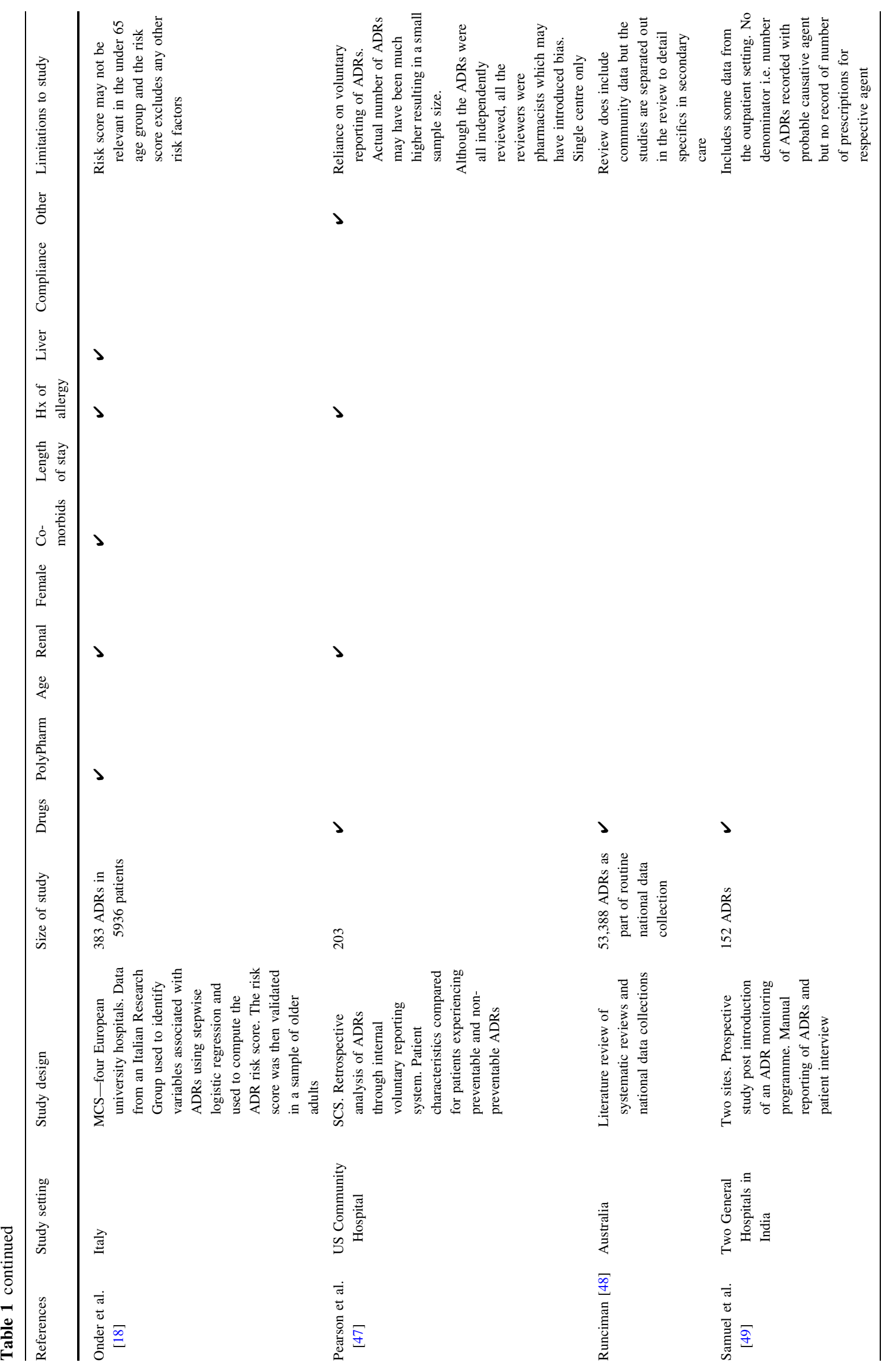




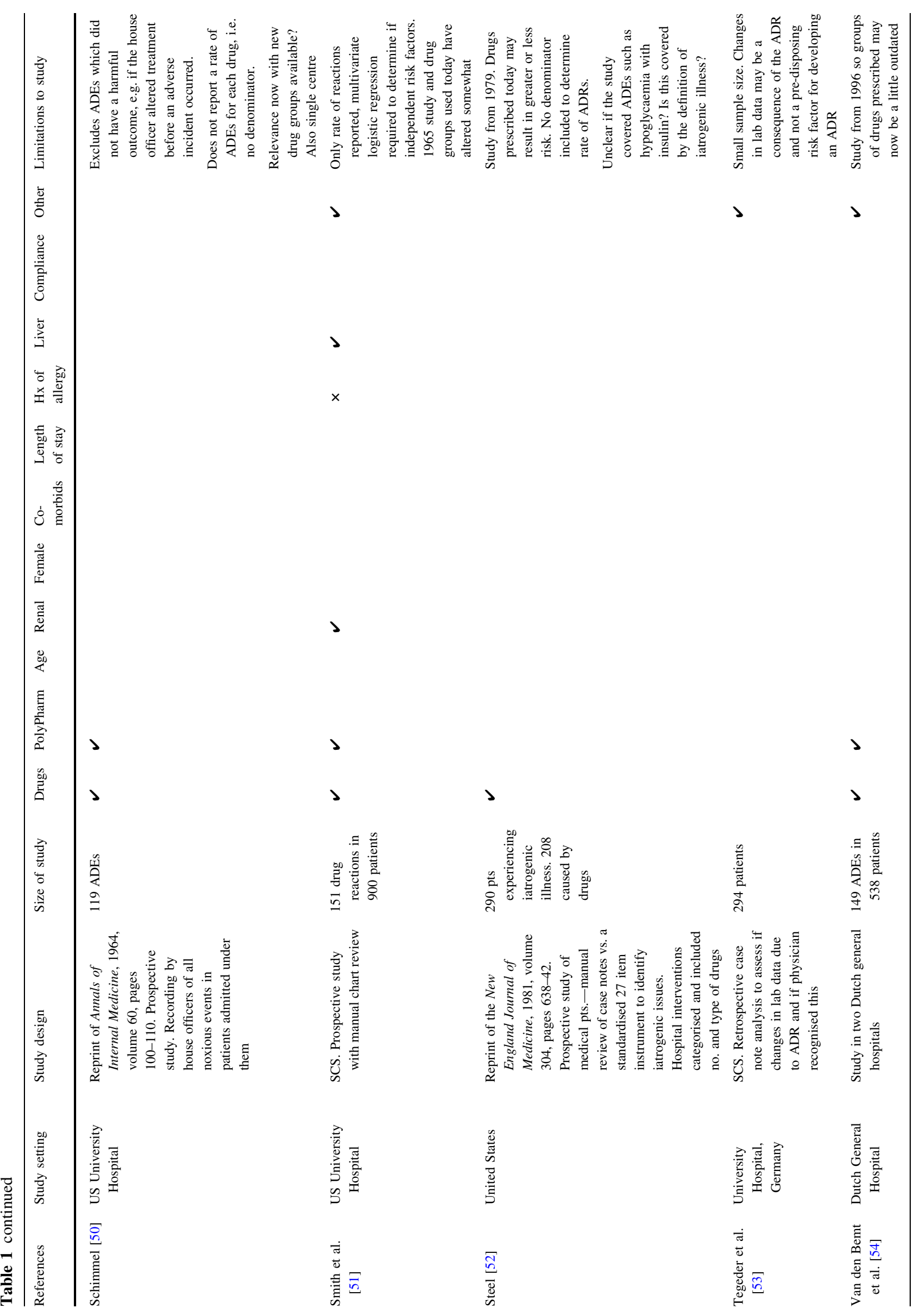




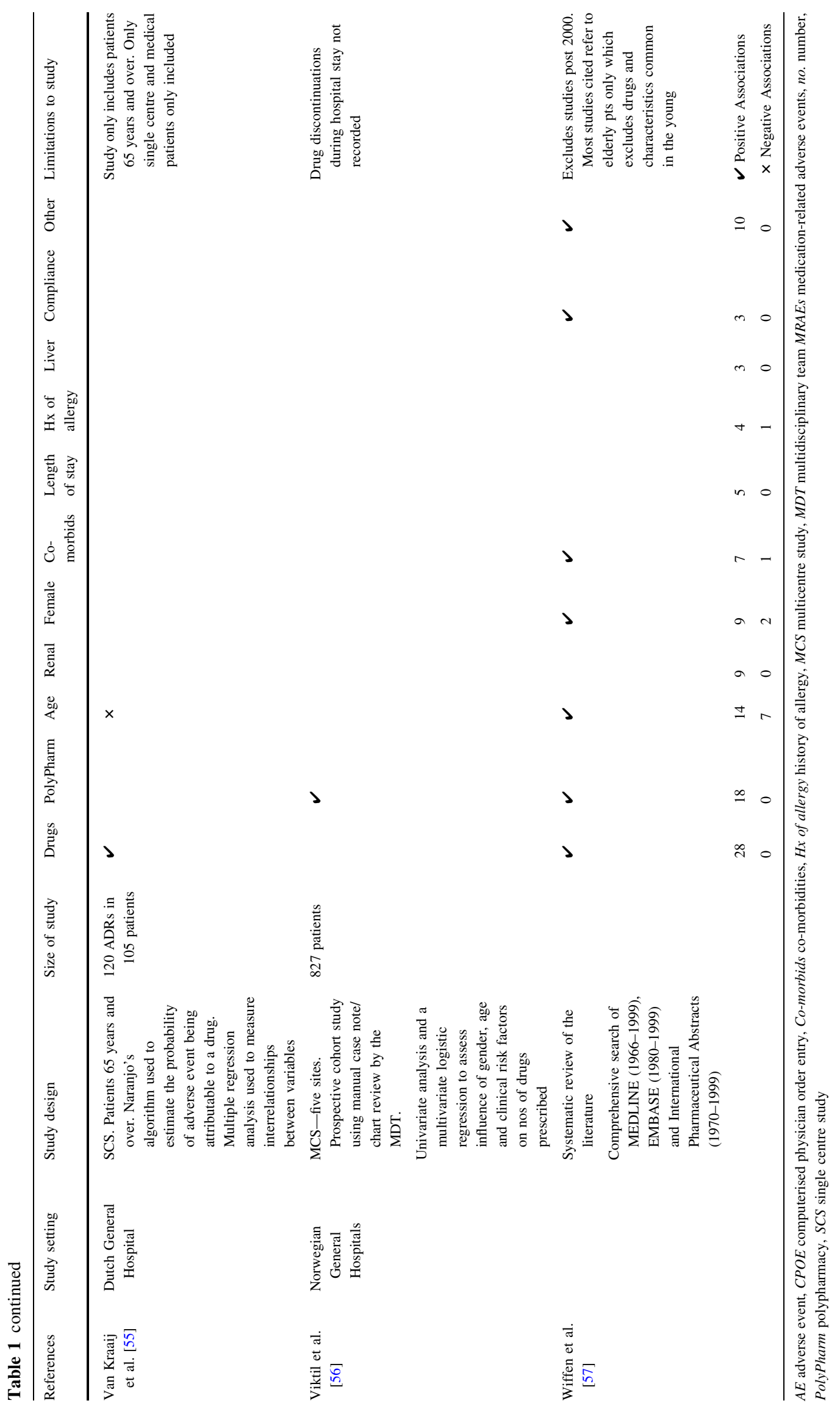


multiple comorbidities, length of patient stay, history of drug allergy or sensitivity, patient compliance issues, and poor liver function.

Table 2 lists the 28 studies that reported that the prescription of certain drugs or classes of drugs were a risk factor in developing a problem with a medicine that may require pharmaceutical intervention. The ten most common classes of drugs reported to be associated with problems in the hospital setting are as follows (in descending order of frequency): intravenous antimicrobials, thrombolytics/anticoagulants, cardiovascular agents, central nervous system (CNS) agents, corticosteroids, diuretics, chemotherapy, insulin/hypoglycaemics, opiates, and anti-epileptics.

\section{Discussion}

A clinical intervention is the process of a pharmacist identifying, and making a recommendation in an attempt to prevent or resolve, a drug-related issue. The definition of an intervention does not include a routine pharmacist review of medication without recommendation for a change in the patient's treatment. Clinical pharmacist interventions are therefore more time consuming and costly to perform than routine pharmacist reviews of a drug chart.

Although there are many research papers that detail the risks associated with DRPs, ADEs, ADRs, drug errors, and MRPs, there is little work exploring the requirement for a pharmacist intervention in these contexts. Pharmacist intervention is appropriate should any of these medicationrelated issues occur and perhaps for others we have yet to identify. This search aimed to identify these risks with a view to future targeting of patients most in need of intervention, thus maximising limited resources.

\subsection{High-Risk Drugs}

The ten risk factors most frequently associated with DRPs, ADEs, ADRs, drug errors, and MRPs are not surprising and yet are poorly documented as such in the literature. The identity of the drugs themselves and the associated class effects are the single largest risk factor and yet it is not possible from the literature to quantify the risk associated with the use of an individual drug or drug class. For example, intravenous antibiotics are the most frequently reported drug class linked with medicines-related problems, while thrombolytics and anticoagulants constitute the second most prevalent group. However, none of the review papers quantify those risks. Further research would be beneficial to identify a risk score for each drug class to facilitate comparisons and measures for prevention. Similarly, there is no information comparing the risks associated with drugs within each class.
The four most commonly named drug groups associated with issues were antimicrobials (mainly intravenous antibiotics), anticoagulants and thrombolytics, cardiovascular drugs, and drugs acting on the CNS. Definitions of these classes of drugs are unclear in almost all of the papers reviewed, making interpretation of the findings and further research problematic. In most papers, the researchers did not consider whether the drug was an independent risk factor. For example, in the case of antimicrobials, none of the researchers considered the possibility that the presence of infection may have been the causative factor leading to an adverse event.

It is important that any conclusions made from this review are interpreted in general medical and surgical settings only. In order to obtain meaningful data, researchers have examined groups of patients taking widely available and frequently prescribed medicines in hospital. None of the review papers reported the frequency of prescribing for a particular drug class, i.e. there was no reported denominator. It is possible that the large number of issues associated with diuretics, for example, is associated with their widespread use. Some newer drugs to the market such as monoclonal antibodies, anti-retrovirals and anti-rejection drugs, which might be expected to be associated with a large number of problems associated with the use of medicines compared with the number of prescriptions, are not included in any of the review papers, and therefore the results do not necessarily mirror alerts for high-risk drugs issued nationally and internationally [13-15].

The present review identified papers within the limitation of date restrictions of the databases searched, which included papers from 1966 to the close of search (July 2013). Despite concerns that this might have a direct impact on the range of drugs identified as high risk, only the inclusion of diuretics as a high-risk category was unexpected. On review of the date of the articles citing diuretics as a high-risk drug category, four of the eight publications were published post 2005; should all papers prior to 2005 in the review be excluded, diuretics would remain as a top 10 high-risk drug.

Since undertaking the present review, another systematic review of high-risk drugs associated with medication errors has been published [17]. The drugs highlighted as high risk in the review are different to those identified in the present review. The review [56] only searched for risks associated with preventable problems, with the assumption that intervention prior to a non-preventable problem would be futile. The present review examines risks associated with both preventable and non-preventable issues with medicines since in cases where problems may not be preventable, prompt recognition and possible removal of the causative agent seems sensible. 


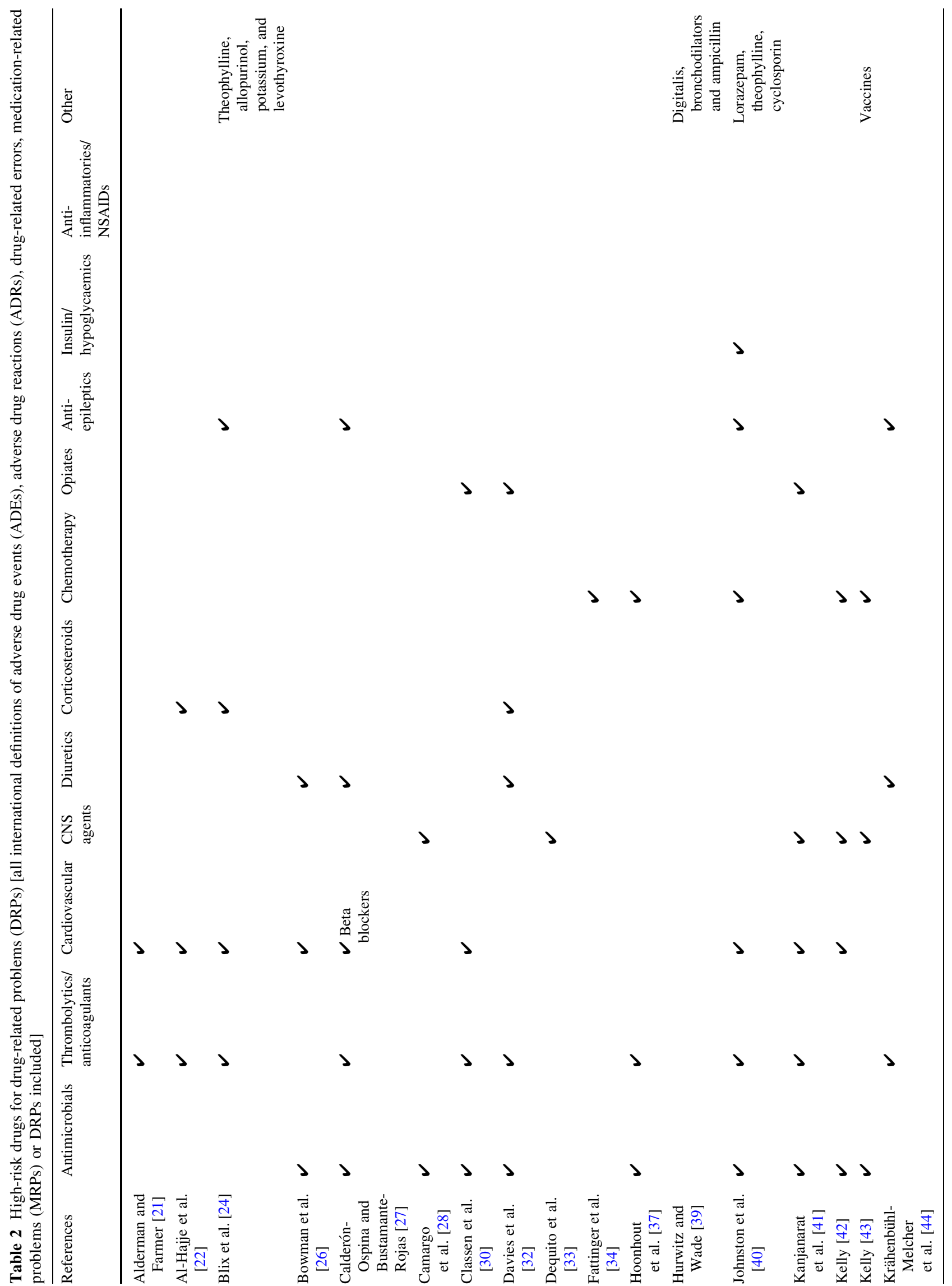




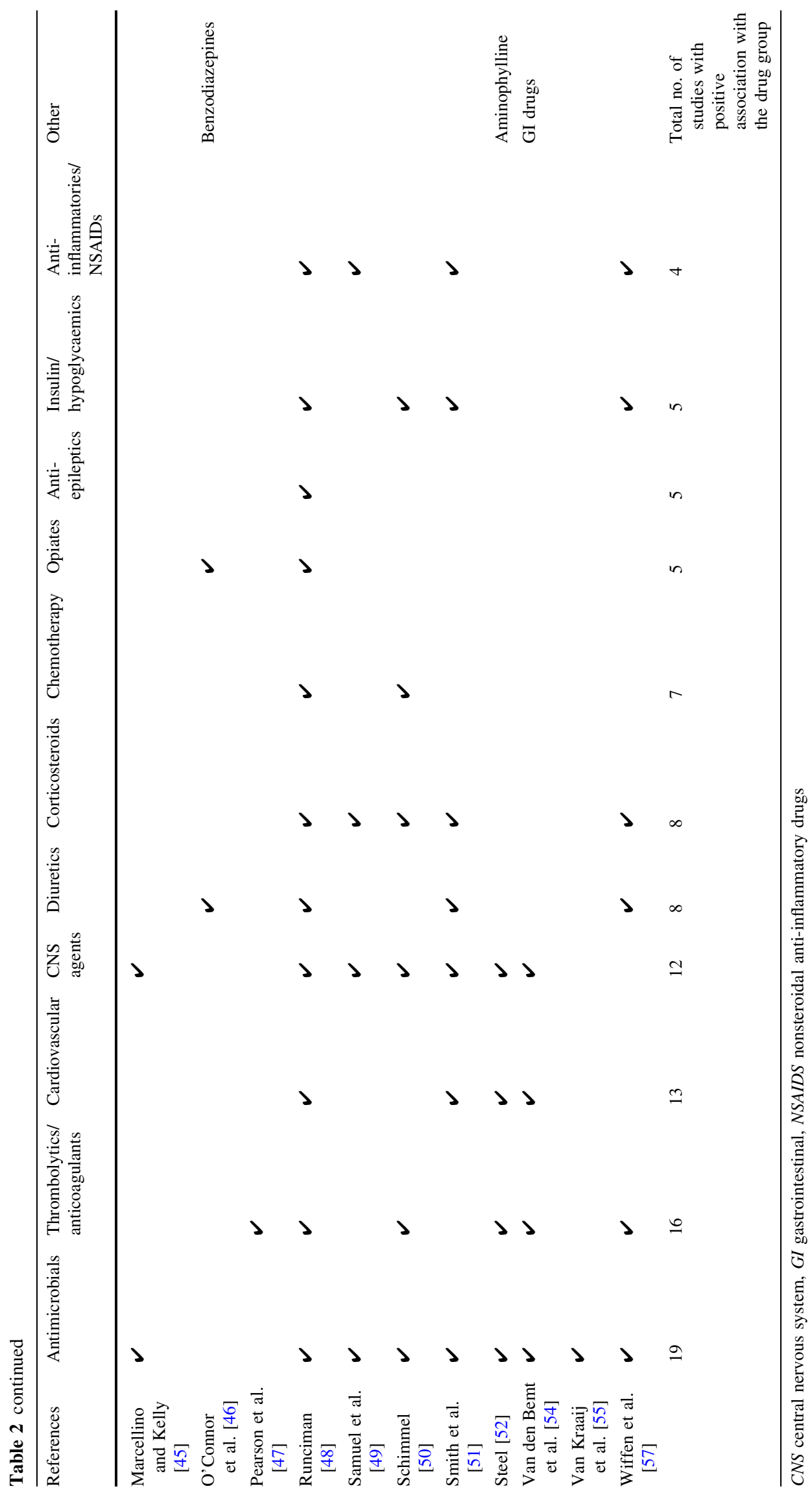


One approach to increasing awareness of the risks associated with individual medicines could be that in the future, all drugs are risk assessed before reaching the market as part of the clinical trial process. Products could be assigned a risk score prior to the issue of market authorisation, using a process similar to that undertaken for intravenous medication under UK NPSA Alert 20 [13]. In light of post-marketing studies and national incident reporting systems, modifications of risk scores could accompany national patient safety alerts.

\subsection{Polypharmacy}

It is widely accepted that polypharmacy has a direct effect on the number of DRPs, ADEs, ADRs, drug errors, and MRPs. This not only seems a logical assumption, but it is also undisputed in the literature where polypharmacy has been shown to be an independent risk factor for the development of problems related to medicines $[18,24,25,28,29,32-34,46,54,56]$. Various definitions of polypharmacy exist, ranging from prescription of three to six or more medicines. However, it is more likely that there is a continuous relationship [56], possibly exponential [57], between the number of drugs taken and the risk of a problem developing.

\subsection{Age}

Older age (definitions vary from 60 years to over 75 years) was reported as a risk factor in 14 studies; however, a further six studies [24, 25, 28, 29, 34, 55] reported that age is not an independent risk factor for medicines-related issues. The six studies that demonstrated that age is unlikely to be an independent risk factor used multi-variant analysis and logistic regression to show that the association of older age with medication problems is more likely to be associated with the increased incidence of multiple comorbidities, multiple medications, poor renal function, and compliance issues in elderly persons rather than a direct association with their age per se. This was supported by a literature review [36] over 20 years ago, which recognised that most studies examining age and ADRs (including all definitions) failed to control for multiple drugs and multiple comorbidities. As the elderly population increases and research in this area continues, it is likely that the risks associated with the use of drugs in old age will become clearer. However, it seems logical that as life expectancy increases, exceeding age 65 years is unlikely to influence the likelihood of suffering an ADR, whereas, the prevailing general state of health will.

One study [31] reported that the age group 18-50 years was a risk factor for ADEs, but it is likely that this was due to the fact that the study group comprised only diabetic patients.

\subsection{Renal Function}

Poor renal function was the fourth most frequently reported risk factor, listed in nine papers [18, 23-25, 35, 44, 46, 47, 51]. However, as long ago as 1966, Smith et al. [51] recognised that this risk factor is only likely to increase the rate of ADRs when using certain groups of drugs that are eliminated renally. However, any patient with poor renal function may potentially be prescribed one of these drugs and, as such, may already be deemed at risk of a problem related to drugs prior to prescription. The recommended dosage or frequency adjustments in renal failure are well documented for affected agents so that this risk may be minimised if appropriately identified. This was supported by Fields et al. [35], who recognised the importance of early estimation of creatinine clearance $(\mathrm{CrCl})$ through computerised order entry to identify renal function as a risk factor for preventable ADEs.

\subsection{Gender}

Female gender is the fifth most frequently reported risk factor for DRPs, ADEs, ADRs, drug errors, and MRPs, with nine papers reporting an association $[25,26$, $31-34,38,44,57]$. However, it is possible that the link with female gender may be weak since one paper demonstrated that gender was not an independent risk factor for ADRs [28], while another reported that ADEs occurred more often in men than in women [40]. However, numbers in the later study [40] were small and most adverse events were due to drug errors, which are unlikely to be affected by the gender of the patient. Further detailed research is required to define the precise relationship.

\subsection{Comorbidities}

Seven papers included multiple comorbidities as a risk factor for problems associated with the use of medicines [21, 24, 26, 28, 30, 44, 55]. However, Camargo et al. [28] used multivariate logistic regression and identified that multiple diagnoses were unlikely to be an independent risk factor for ADRs. It is possible that the increased number of medicines taken by patients with multiple comorbidities could have a bearing on the number of problems experienced by patients. Conversely, it is also possible that a patient's susceptibility to ADRs is increased by their poor overall health and that drug metabolism may be affected by their condition or additional unknown factors. It would be advisable for more research to be carried out in this area.

\subsection{Length of Stay}

Length of hospital stay was also reported as a risk factor $[26,28-30,33]$. This seems a logical connection in that any 
adverse event (drug related or otherwise) is more likely to occur the longer the patient is observed, which in the case of hospital inpatients, would be dependent on their length of stay. However, one paper [28] reported that there is an association with follow-up period (period of time as an inpatient after an ADR). In this case, it is possible that the occurrence of an ADR caused the increase in length of stay through treatment failure, drug toxicity, or other factors. None of the review papers reported that patients were more likely statistically to experience a problem the longer the patient stayed in hospital, i.e. the intra-patient risk at any point in time does not increase with the length of inpatient stay. However, it is also likely that patients who have longer hospital stays suffer from complex conditions or are more unwell, making them more susceptible to DRPs throughout their stay; under such circumstances, length of stay is not an independent risk factor.

\subsection{History of Allergy and Compliance Issues}

Other risk factors for DRPs, ADEs, ADRs, drug errors, and MRPs included in the literature were a previous history of allergy or ADR and compliance issues, which were listed as risk factors in four [18, 24, 38, 47] and three [24, 29, 57] papers, respectively. Patients who may have a genetic predisposition to ADRs or who display atopic characteristics may be more likely to experience ADRs. One paper [51] noted that although there was not an overall increase of ADRs in this group, there was an increase in allergic reactions.

Compliance issues included assumed non-compliance, low cognition, and other factors affecting patients taking their medicines such as alcohol abuse and swallowing difficulties. Such barriers to compliance intuitively would predispose patients to problems with medication regimens.

\subsection{Liver Function}

The association of deteriorating liver function with DRPs, ADEs, ADRs, drug errors, and MRPs is less well documented. Only three papers [18, 24, 51] list deteriorating liver function as a risk factor. In an analogous situation to renal impairment, poor liver function is likely to only be associated with an increased risk when certain drugs are used, i.e. those whose elimination or distribution is hepatic or affected by the reduction in protein metabolism, which accompanies deterioration of liver function. Again this relationship was recognised by Smith et al. [51], who noted in his study that although the overall rate of ADRs was not increased by decreasing liver function, the rate for certain groups of drugs was increased slightly.

Drug management in hepatic failure generally differs to therapy in renal failure. Often the risks of hepatotoxicity drive the decision to treat with a drug or not, in contrast to dosage or frequency adjustments required to avoid immediate toxicity or treatment failure encountered in renal failure. Prescribers often only have one of two options when considering a drug for use in liver failure- "To use or not to use?"- -essentially a $50 \%$ chance of making the correct decision and avoiding toxicity that may (or may not) result in an adverse event related to the use of medicines. The likelihood of ADEs in patients with renal failure as opposed to liver failure seems much greater owing to errors in prescribing. These issues are compounded in renal failure owing to drug accumulation or treatment failure as $\mathrm{CrCl}$ reduces.

\subsection{Other Risk Factors}

Other risk factors that were uniquely identified (and were therefore not tabulated as top 10 risk factors in this review) included admission to a medical ward [32], geriatric ward, rheumatology ward or gastroenterology ward [33], source of admission (e.g. from home, general practitioner, clinic, etc.) [40], insurance class (US) [40], infection [51], changes in patient's biochemical/haematological parameters [53], new drug initiation in hospital [54], single marital status [31], use of drugs with a narrow therapeutic index [44], and TDM requirement in the absence of a pharmacokinetics service [47]. Since these associations were only reported in single studies, there may have been explanations for the reported risk factors. It seems likely that drugs with a narrow therapeutic index or requiring TDM are indeed generic risk factors in all specialities and that starting a new drug in any setting poses a risk owing to drug error or poor compliance. However, it is less obvious that factors such as single marital status are independent risk factors for DRPs. Perhaps married patients may be older and their lifestyle more predictable, providing a supportive environment for improved compliance.

\subsection{Limitations to the Review}

The present review methods relied on the use of a number of electronic databases, all of which used English as the primary language, and all of the journals searched were publications in the English language. As a consequence, although the databases included citations from international journals, it is likely that there is a bias towards publications in English and that other work, in particular from the Far East, may have been overlooked. However, the review did not exclude publications from non-English outputs. When the full texts were received, 44 citations were identified from cross-referencing. It was found that 30 of these papers were available through databases listed in the review, indicating that the online database search had 
not captured all relevant papers. However, these databases have been rechecked using various other combinations of the search terms listed in an attempt to confirm that no other papers remain. In particular, cross-referencing identified a number of older articles that have been included in this review but whose significance may be debatable owing to differences in drug treatments available historically.

No outputs listing risk factors requiring intervention by a pharmacist were detected using these methods. It is possible that research into pharmacist interventions would assume a direct correlation between pharmacist intervention and an ADE. Researchers may deem it more appropriate to assess risk factors leading to the latter since the presence of an adverse event indicates that either there has been no preventative intervention or an intervention has been unsuccessful in prevention of the adverse event. Whichever is the case, without a proven correlation between pharmaceutical intervention and the outcome of an adverse event, research methodology may be better directed at risk factors leading to adverse events caused by medicines use.

Similarly, as research into pharmacist interventions is more likely to be carried out by pharmacists themselves who already target patients perceived to be at risk, this may result in bias. Intervention research is more likely to be targeted at those areas pharmacists may be missing, i.e. actual reported problems associated with medicines rather than pharmacist interventions, i.e. the near miss. Pharmacists may wish to determine whether problems are preventable, non-preventable, or partially preventable through pharmaceutical intervention before targeting clinical pharmacy services to patients with risk factors for medicines-related issues. Certainly, research in this area is lacking and has resulted in difficulties in quantifying the worth of clinical pharmacy services.

\section{Conclusions}

Review of the literature found 38 papers that detailed ten measurable risk factors linked with DRPs in hospital inpatients that were identified in the literature by more than one primary research article. DRPs included all international definitions of ADEs, ADRs, DRPs, and MRPs. No papers were detected that identified risk factors for pharmacist interventions. There is a need for studies to be carried out in this area in order that clinical pharmacy services may be directed appropriately. Although risks associated with incidents and issues that arise from treatment with medication may be similar, it is likely that there are additional risk factors that cause pharmacists to intervene that are not associated with the drug treatment in use. For example, a patient may not be taking any medication at all and raise a question regarding lifestyle choice or dietary advice. This review showed that research into activity carried out by clinical pharmacists in hospital is lacking.

However, all of the potential risk factors identified from this review may be identified from most patients' records on admission to hospital. It is hoped that these risk factors may be used to indicate patients most at risk, with a view to targeting pharmaceutical input in order to minimise medicines-related problems. With the advent of CDS systems, quantifying such risk factors could enable the restructure of clinical pharmacy services into a model targeting patients most at risk in real time rather than the ward/unit in which they are located, which is most often the case in the UK.

Potential risk factors include prescription of certain drugs or classes of drugs, polypharmacy, elderly patients (defined as over 60-75 years or older), female gender, poor renal function, the presence of multiple comorbidities, length of patient stay, history of drug allergy or sensitivity, patient compliance issues, and poor liver function.

Hospital use of medications that are associated with a high risk include antimicrobials (intravenous antibiotics), anticoagulants and thrombolytics, cardiovascular drugs, and drugs acting on the CNS. More research is required to ensure that newer drug classes are included in research into risks associated with the use of medicines and whether the risks associated with the use of high-risk drugs are preventable.

Acknowledgments The authors would like to acknowledge Sarah Thomas (MRPharmS PG Dip), NIHR Doctoral Research Fellow, School of Clinical and Experimental Medicine, College of Medical and Dental Sciences, University of Birmingham, for her assistance with validation of review results.

\section{Compliance with Ethical Standards}

Conflict of interest Emma Suggett B.Sc. Hons, MRPharmS, ClinDipPharm and John Marriott, B.Sc. Hons, Ph.D., Professor of Clinical Pharmacy have no conflicts of interest to declare.

Funding Author Emma Suggett is currently employed by the University Hospitals Birmingham NHS Foundation Trust (UHBFT). UHBFT have sponsored the author to undertake a doctorate programme in Pharmacy (DPharm) with the University of Birmingham. This systematic review was undertaken as part of the author's thesis, and John Marriott is the supervisor.

Open Access This article is distributed under the terms of the Creative Commons Attribution-NonCommercial 4.0 International License (http://creativecommons.org/licenses/by-nc/4.0/), which permits any noncommercial use, distribution, and reproduction in any medium, provided you give appropriate credit to the original author(s) and the source, provide a link to the Creative Commons license, and indicate if changes were made. 


\section{References}

1. Department of Health. Quality, innovation, productivity and prevention (QIPP). [Internet]. http://www.dh.gov.uk/en/ Healthcare/Qualityandproductivity/QIPP/index.htm.

2. Lazarou J, Pomeranz BH, Corey PN. Incidence of adverse drug reactions in hospitalized patients. JAMA J Am Med Assoc. 1998;279:1200-5.

3. Avery AJ, Rodgers S, Cantrill JA, et al. A pharmacist-led information technology intervention for medication errors (PINCER): a multicentre, cluster randomised, controlled trial and cost-effectiveness analysis. Lancet. 2012;379:1310-9.

4. Carter BL, Malone DC, Billups SJ, et al. Interpreting the findings of the IMPROVE study. Am J Health-Syst Pharm AJHP Off J Am Soc. Health-Syst Pharm. 2001;58:1330-7.

5. Fick DM, Cooper JW, Wade WE, et al. Updating the Beers criteria for potentially inappropriate medication use in older adults: results of a US consensus panel of experts. Arch Intern Med. 2003;163:2716-24.

6. Lam MP, Cheung BM. The use of STOPP/START criteria as a screening tool for assessing the appropriateness of medications in the elderly population. Expert Rev Clin Pharmacol. 2012;5:187-97.

7. Masica AL, Touchette DR, Dolor RJ, et al. Evaluation of a medication therapy management program in Medicare beneficiaries at high risk of adverse drug events: study methods. Adv Patient Saf N Dir Altern Approaches. 2008;4:8-34.

8. Gordon KJ, Smith FJ, Dhillon S. The development and validation of a screening tool for the identification of patients experiencing medication-related problems. Int J Pharm Pract. 2005;13:187-93.

9. Meyer-Massetti C, Cheng CM, Schwappach DLB, et al. Systematic review of medication safety assessment methods. Am J Health Syst Pharm. 2011;68:227-40.

10. Neubert A, Dormann H, Prokosch H-U, et al. E-pharmacovigilance: development and implementation of a computable knowledge base to identify adverse drug reactions. Br J Clin Pharmacol. 2013;76:69-77.

11. Rommers MK, Zegers MH, De Clercq PA, et al. Development of a computerised alert system, ADEAS, to identify patients at risk for an adverse drug event. Qual Saf Health Care. 2010;19:e35.

12. Silverman JB, Stapinski CD, Huber C, et al. Computer-based system for preventing adverse drug events. Am J Health-Syst Pharm AJHP Off J Am Soc Health-Syst Pharm. 2004;61:1599-603.

13. National Patient Safety Agency (NPSA) Alerts 2013 [Internet]. [cited 2014 Jan 12]. http://www.nrls.npsa.nhs.uk/resources/type/ alerts.

14. Institute for Safe Medicines Practices (ISMP) Medication Safety Tools and Resources [Internet]. [cited 2014 Jun 2]. http://www. ismp.org/tools/.

15. Australian Commission on Safety and Quality in Health Care (ACSQHC) Alerts [Internet]. [cited 2014 Jun 2]. http://www. safetyandquality.gov.au/our-work/medication-safety/medicationalerts/.

16. Garrett D. MUSN. An overview of the medication safety team priorities at NHS England: medicines thermometer. 2013 [Internet]. [cited 2016 July 11]. http://www.google.co.uk/url?sa=t\&rct $=\mathrm{j} \& \mathrm{q}=\& \mathrm{esrc}=\mathrm{s} \&$ source $=$ web $\& \mathrm{~cd}=2 \& \mathrm{cad}=\mathrm{rja} \& u a c t=8 \& \mathrm{ved}=0 \mathrm{ah}$ UKEwjxkOm7n-zNAhVMBMAKHfa9AfEQFggiMAE\&url=http $\% 3 \mathrm{~A} \% 2 \mathrm{~F} \% 2 \mathrm{Fwww}$.medicinesresources.nhs.uk\%2Fen\%2FDown load\%2F\%3Ffile\%3DMDs3NDc3MzY7L3VwbG9hZC9kb2N1b WVudHMvQ29tbXVuaXRpZXMvU1BTX0VfU0VfRW5nbGFu ZC9QcmVzb19NVVNOXzZKdW4xM19NZWRzX3RoZXJtb21 ldGVyX0RHLnBkZg_.ppdf\&usg=AFQjCNGmNMhjBculv4n0E-Uxwhh5DOriA\&bvm=bv.126130881,d.ZGg
17. Saedder EA, Brock B, Nielsen LP, et al. Identifying high-risk medication: a systematic literature review. Eur J Clin Pharmacol. 2014;70:637-45.

18. Onder G, Petrovic M, Tangiisuran B, et al. Development and validation of a score to assess risk of adverse drug reactions among in-hospital patients 65 years or older: the GerontoNet ADR risk score. Arch Intern Med. 2010;170:1142-8.

19. Moher D, Liberati A, Tetzlaff J, et al. Preferred reporting items for systematic reviews and meta-analyses: the PRISMA statement. BMJ. 2009;339:b2535-b2535.

20. Richardson WS, Wilson MC, Nishikawa J, et al. The well-built clinical question: a key to evidence-based decisions. ACP J Club. 1995;123:A12-3.

21. Alderman CP, Farmer C. A brief analysis of clinical pharmacy interventions undertaken in an Australian teaching hospital. J Qual Clin Pract. 2001;21:99-103.

22. Al-Hajje AH, Atoui F, Awada S, et al. Drug-related problems identified by clinical pharmacist's students and pharmacist's interventions. Ann Pharm Fr. 2012;70:169-76.

23. Bates DW, Miller EB, Cullen DJ, et al. Patient risk factors for adverse drug events in hospitalized patients. ADE Prevention Study Group. Arch Intern Med. 1999;159:2553-60.

24. Blix HS, Viktil KK, Reikvam A, et al. The majority of hospitalised patients have drug-related problems: results from a prospective study in general hospitals. Eur J Clin Pharmacol. 2004;60:651-8.

25. Bowman L, Carlstedt BC, Hancock EF, et al. Adverse drug reaction (ADR) occurrence and evaluation in elderly inpatients. Pharmacoepidemiol Drug Saf. 1996;5:9-18.

26. Bowman L, Carlstedt BC, Black CD. Incidence of adverse drug reactions in adult medical inpatients. Can J Hosp Pharm. 1994;47:209.

27. Calderón-Ospina C, Bustamante-Rojas C. The DoTS classification is a useful way to classify adverse drug reactions: a preliminary study in hospitalized patients: evaluation of adverse drug reactions. Int J Pharm Pract. 2010;18:230-5.

28. Camargo AL, Ferreira MBC, Heineck I. Adverse drug reactions: a cohort study in internal medicine units at a university hospital. Eur J Clin Pharmacol. 2006;62:143-9.

29. Carbonin P, Pahor M, Bernabei R, et al. Is age an independent risk factor of adverse drug reactions in hospitalized medical patients? J Am Geriatr Soc. 1991;39:1093-9.

30. Classen DC, Pestotnik SL, Evans RS, et al. Computerized surveillance of adverse drug events in hospital patients. JAMA. 1991;266:2847-51.

31. Claydon-Platt K, Manias E, Dunning T. Medication-related problems occurring in people with diabetes during an admission to an adult teaching hospital: a retrospective cohort study. Diabetes Res Clin Pract. 2012;97:223-30.

32. Davies EC, Green CF, Taylor S, et al. Adverse drug reactions in hospital in-patients: a prospective analysis of 3695 patient-episodes. PLoS One. 2009;4:e4439.

33. Dequito AB, Mol PGM, van Doormaal JE, et al. Preventable and non-preventable adverse drug events in hospitalized patients: a prospective chart review in the Netherlands. Drug Saf Int J Med Toxicol Drug Exp. 2011;34:1089-100.

34. Fattinger K, Roos M, Vergères $P$, et al. Epidemiology of drug exposure and adverse drug reactions in two Swiss departments of internal medicine. Br J Clin Pharmacol. 2000;49:158-67.

35. Fields W, Tedeschi C, Foltz J, et al. Reducing preventable medication safety events by recognizing renal risk. Clin Nurse Spec CNS. 2008;22:73-80.

36. Gurwitz JH, Avorn J. The ambiguous relation between aging and adverse drug reactions. Ann Intern Med. 1991;114:956-66.

37. Hoonhout LH, de Bruijne MC, Wagner C, et al. Nature, occurrence and consequences of medication-related adverse events 
during hospitalization: a retrospective chart review in the Netherlands. Drug Saf. 2010;33:853-64.

38. Hurwitz N. Predisposing factors in adverse reactions to drugs. $\mathrm{Br}$ Med J. 1969;1:536-9.

39. Hurwitz N, Wade OL. Intensive hospital monitoring of adverse reactions to drugs. Br Med J. 1969;1:531.

40. Johnston PE, France DJ, Byrne DW, et al. Assessment of adverse drug events among patients in a tertiary care medical center. Am J Health-Syst Pharm AJHP Off J Am Soc Health-Syst Pharm. 2006;63:2218-27.

41. Kanjanarat P, Winterstein AG, Johns TE, et al. Nature of preventable adverse drug events in hospitals: a literature review. Am J Health-Syst Pharm AJHP Off J Am Soc Health-Syst Pharm. 2003;60:1750-9.

42. Kelly WN. Potential risks and prevention, part 1: fatal adverse drug events. Am J Health Syst Pharm. 2001;58:1317-24.

43. Kelly WN. Potential risks and prevention, part 2: drug-induced permanent disabilities. Am J Health Syst Pharm. 2001;58:1325-9.

44. Krähenbühl-Melcher A, Schlienger R, Lampert M, et al. Drugrelated problems in hospitals: a review of the recent literature. Drug Saf Int J Med Toxicol Drug Exp. 2007;30:379-407.

45. Marcellino K, Kelly WN. Potential risks and prevention, part 3: drug-induced threats to life. Am J Health Syst Pharm. 2001;58:1399-405.

46. O'Connor MN, Gallagher P, Byrne S, et al. Adverse drug reactions in older patients during hospitalisation: are they predictable? Age Ageing. 2012;41:771-6.

47. Pearson TF, Pittman DG, Longley JM, et al. Factors associated with preventable adverse drug reactions. Am J Hosp Pharm. 1994;51:2268-72.
48. Runciman WB. Adverse drug events and medication errors in Australia. Int J Qual Health Care. 2003;15:49i-59i.

49. Samuel SA, Rajendran SD, Ebenezzar S, et al. Surveillance of adverse drug reactions at two multidisciplinary hospitals and an outpatient specialty clinic in India. Int $\mathbf{J}$ Pharm Pract. 2002;10:115-20.

50. Schimmel EM. The hazards of hospitalization. Qual Saf Health Care. 2003;12:58-63.

51. Smith JW, Seidl LG, Cluff LE. Studies on the epidemiology of adverse drug reactions V. Clinical factors influencing susceptibility. Ann Intern Med. 1966;65:629-40.

52. Steel K. Iatrogenic illness on a general medical service at a university hospital. Qual Saf Health Care. 2004;13:76-80.

53. Tegeder I, Levy M, Muth-Selbach U, et al. Retrospective analysis of the frequency and recognition of adverse drug reactions by means of automatically recorded laboratory signals. Br J Clin Pharmacol. 1999;47:557-64.

54. Van den Bemt P, Egberts ACG, Lenderink AW, et al. Risk factors for the development of adverse drug events in hospitalized patients. Pharm World Sci. 2000;22:62-6.

55. Van Kraaij DJ, Haagsma CJ, Go IH, et al. Drug use and adverse drug reactions in 105 elderly patients admitted to a general medical ward. Neth J Med. 1994;44:166.

56. Viktil KK, Blix HS, Moger TA, et al. Polypharmacy as commonly defined is an indicator of limited value in the assessment of drug-related problems. Br J Clin Pharmacol. 2007;63:187-95.

57. Wiffen $\mathrm{P}$, Gill M, Edwards J, et al. Adverse drug reactions in hospital patients: a systematic review of the prospective and retrospective studies. Bandolier Extra. 2002;101:1-16. 